\title{
Supporting Information: \\ Insights into the Polymorphic Structures and Enantiotropic Layer Slip Transition in Paracetamol Form III from Enhanced Molecular Dynamics
}

Richard S. Hong, ${ }^{\mathrm{a}, \mathrm{b}}$ Eric J. Chan, ${ }^{\mathrm{a}}$ Leslie Vogt-Maranto, ${ }^{\mathrm{a}}$ Alessandra Mattei, ${ }^{\mathrm{b}}$ Ahmad Y. Sheikh, ${ }^{\mathrm{b}}$ Mark E. Tuckerman ${ }^{\mathrm{a}, \mathrm{c}, \mathrm{d} *}$

${ }^{a}$ Department of Chemistry, New York University, New York City, New York 10003, United States

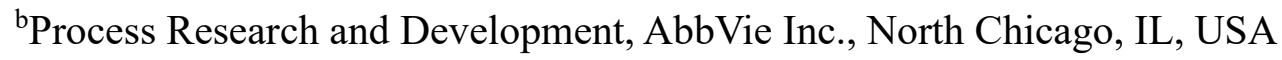
'Courant Institute of Mathematical Sciences, New York University, New York, NY 10012, USA ${ }^{\mathrm{d}}$ NYU-ECNU Center for Computational Chemistry at NYU Shanghai, 3663 Zhongshan Road North, Shanghai 200062, China

Table of Contents

Crystal-AFED Equations of Motion ............................................................................................... 2

Comparison of Crystal-AFED vs NPTF simulation Free Energies:............................................. 3

Full Histograms of Angles Throughout the Trajectory ........................................................................ 4

Comparison Free Energy Curves vs Larger Super-Cells ................................................................ 6

Scatter Plot of Neighboring Layer Pair Displacements at 100K.................................................. 7

Scatter Plot of Neighboring Layer Pair Displacements at 300K: ................................................. 9

Displacement Scatter Plots for 2x2x4 Super-Cell with many more layers for Non-Neighboring

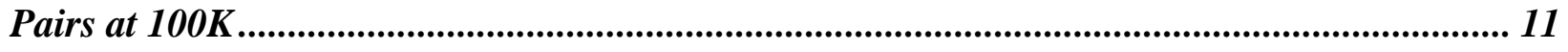

Displacement Scatter Plots for 2x2x4 Super-Cell with many more layers for Non-Neighboring Pairs at 300K ........................................................................................................................ 12

Determination of Layer Slip Measure................................................................................. 13

Simulation Free Energy Comparisons Between III-o Starting Structure and III-m Starting Structure ..................................................................................................................................... 14 


\section{Crystal-AFED Equations of Motion}

Consider a molecular crystal with $\mathrm{N}$ atoms that have the respective coordinates of $\mathbf{r}_{1} \ldots \mathbf{r}_{\mathbf{N}}$ and respective momenta of $\mathbf{p}_{1} \ldots . . \mathbf{p}_{\mathbf{N}}$ interacting with a potential $\mathrm{U}\left(\mathbf{r}_{1} \ldots \mathbf{r}_{\mathbf{N}}\right)$, where the periodic supercell of the system is described by vectors $\mathbf{a}, \mathbf{b}$, and $\mathbf{c}$ where the cell volume of the super cell is described by $\mathrm{V}=\operatorname{det}(\mathbf{h})$.

From the predefined variables, the equations of motion of Crystal-AFED are as follows:

$$
\begin{gathered}
\dot{\mathbf{r}}_{\mathrm{i}}=\frac{\mathbf{p}_{\mathrm{i}}}{\mathrm{m}_{\mathrm{i}}}+\frac{\mathbf{p}_{\mathrm{g}}}{\mathrm{W}} \mathbf{r}_{\mathrm{i}} \\
\dot{\mathbf{p}_{\mathbf{i}}}=\boldsymbol{F}_{i}-\frac{\boldsymbol{p}_{g}}{W} \boldsymbol{p}_{i}-\frac{1}{N_{f}} \frac{\operatorname{Tr}\left[\boldsymbol{p}_{g}\right]}{W} \boldsymbol{p}_{i}+\boldsymbol{H}_{i}(T) \\
\dot{\mathbf{h}}=\frac{\mathbf{p}_{\mathbf{g}} \mathbf{h}}{\mathrm{W}} \\
\dot{\mathbf{p}_{\mathbf{g}}}=\operatorname{det}(\mathbf{h})\left[\mathbf{P}^{\mathrm{int}}-\mathrm{PI}\right]+\frac{1}{\mathrm{~N}_{\mathrm{f}}} \sum_{i=1}^{N} \frac{\boldsymbol{p}_{i}^{2}}{m_{i}} \boldsymbol{I}+\boldsymbol{H}\left(T_{h}\right)
\end{gathered}
$$

$\mathrm{F}_{\mathrm{i}}$ is the force on particle $\mathrm{i}$ including any constraints, where $F_{i}=-\frac{d U}{d \boldsymbol{r}_{\boldsymbol{i}}}+\boldsymbol{f}_{i}^{(\text {constr })}$.

The variable $p_{g}$ is a $3 \times 3$ matrix that acts as a barostat to ensure the internal pressure tensor of the system $\boldsymbol{P}^{i n t}=V^{-1} \sum_{i=1}^{N}\left[\boldsymbol{p}_{\boldsymbol{i}} \boldsymbol{p}_{\boldsymbol{i}}+\boldsymbol{r}_{\boldsymbol{i}} \boldsymbol{F}_{\boldsymbol{i}}\right]$ throughout the trajectory averages out to the defined experimental pressure.

$\boldsymbol{H}_{\boldsymbol{i}}(T)$ indicates that there is a heat bath coupling at $\mathrm{T}$ where each degree of freedom is coupled to its own thermostat.

$\mathrm{W}$, as stated in the main text, represents an effective mass that is assigned to the barostat to ensure adiabatic de-coupling of the $3 \times 3 \mathrm{~h}$ matrix, represented by $\mathrm{h}$, to the system.

$\boldsymbol{H}\left(T_{h}\right)$ is a matrix of heat baths assigned to the barostat to ensure the barostat has a temperature of the assigned fictitious cell temperature, $T_{h}$, with $T_{h} \gg \mathrm{T}$, where $\mathrm{T}$ is the molecular temperature of the system being studied.

The equations of motion from Crystal-AFED were used to determine the Gibbs free energy surface by determining the probability distribution of the cell matrix, $P_{a b d}\left(\boldsymbol{h}, T, T_{-} h\right)$ where $G(\boldsymbol{h}, T)=-k_{b} T_{h} \ln P_{a d b}(\boldsymbol{h}, T, T h)$. In this study, the Gibbs free energy surfaces were determined through the probability distribution of the cell alpha angle. 


\section{Comparison of Crystal-AFED vs NPTF simulation Free Energies:}

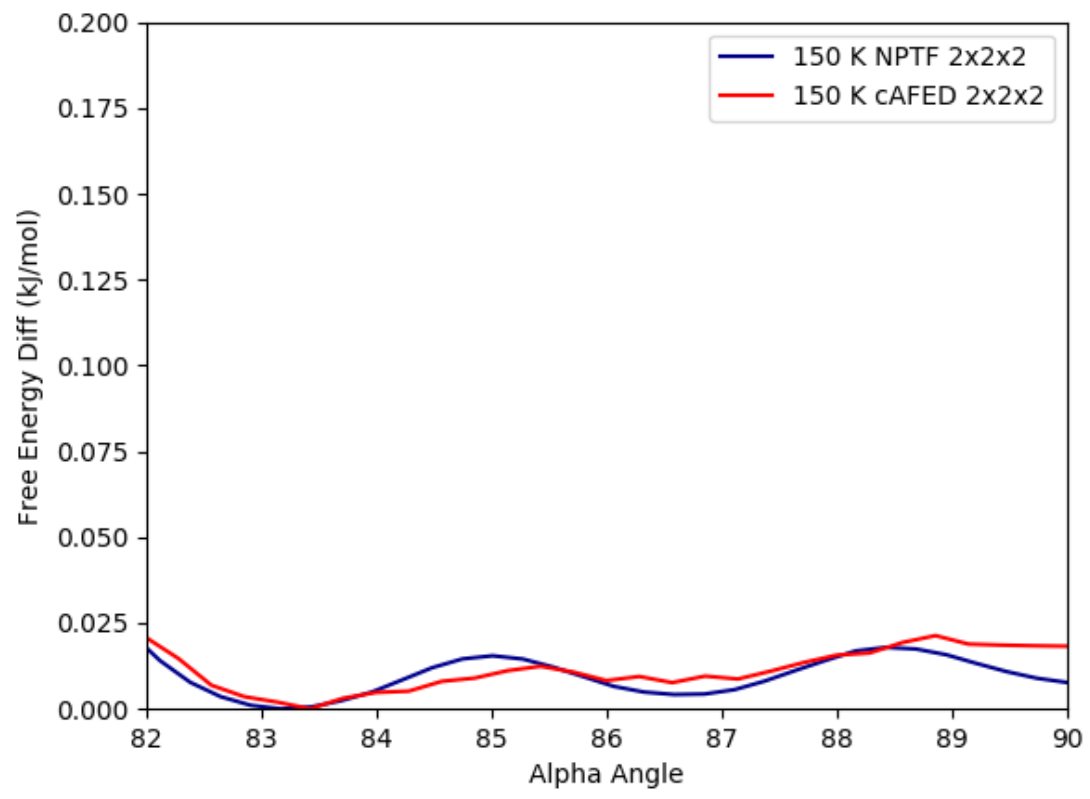

Figure S1. Comparison of the folded free energy profile of a 150K 30 ns NPTF run vs $150 \mathrm{~K} 20$ ns Crystal-AFED run at $\mathrm{Th}=300 \mathrm{~K}, \mathrm{Tau}=2000 \mathrm{ps}$

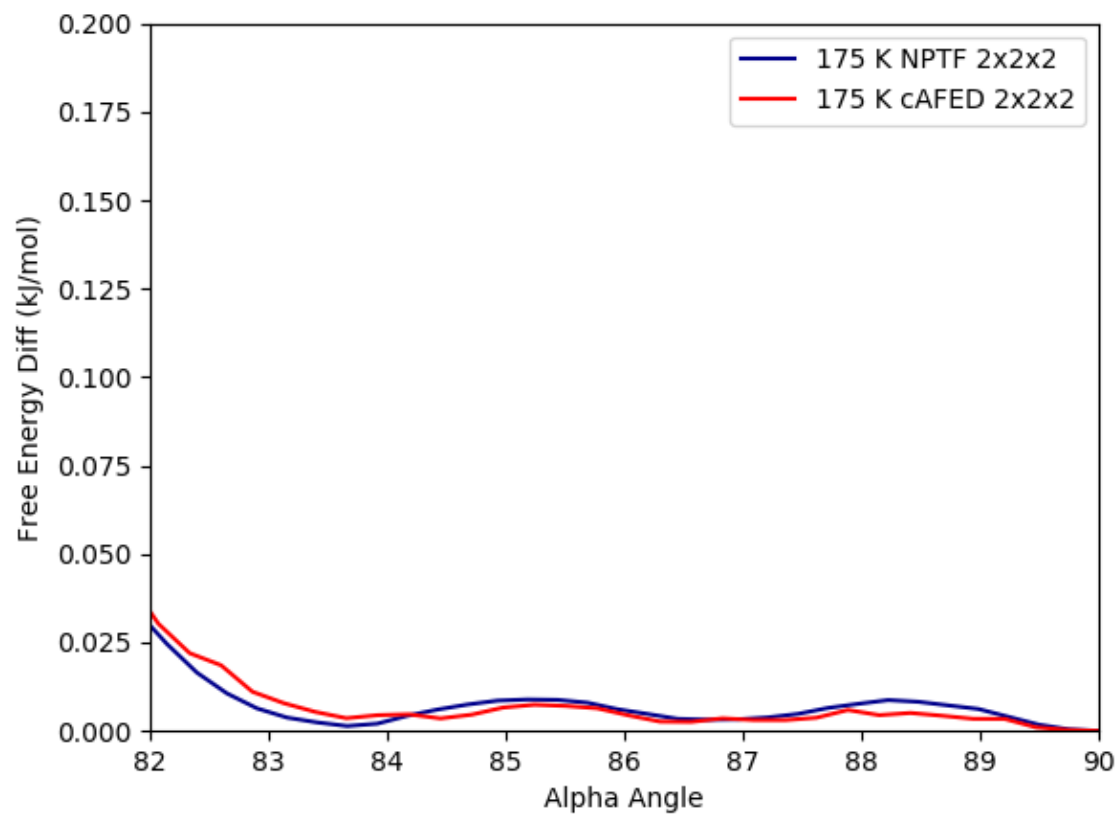

Figure S2. Comparison of the folded free-energy profile of a $175 \mathrm{~K}$ NPTF run vs $175 \mathrm{~K}$ CrystalAFED run at $\mathrm{Th}=300 \mathrm{~K}$, Tau $=2000 \mathrm{ps}$ 
Full Histograms of Angles Throughout the Trajectory


Figure S3 - Distribution of Alpha Angles through out the trajectory for Crystal-AFED simulations at a) $50 \mathrm{~K}$, b) $75 \mathrm{~K}$, c) $100 \mathrm{~K}$, d) $125 \mathrm{~K}$, e) $150 \mathrm{~K}$, f) $175 \mathrm{~K}$ 

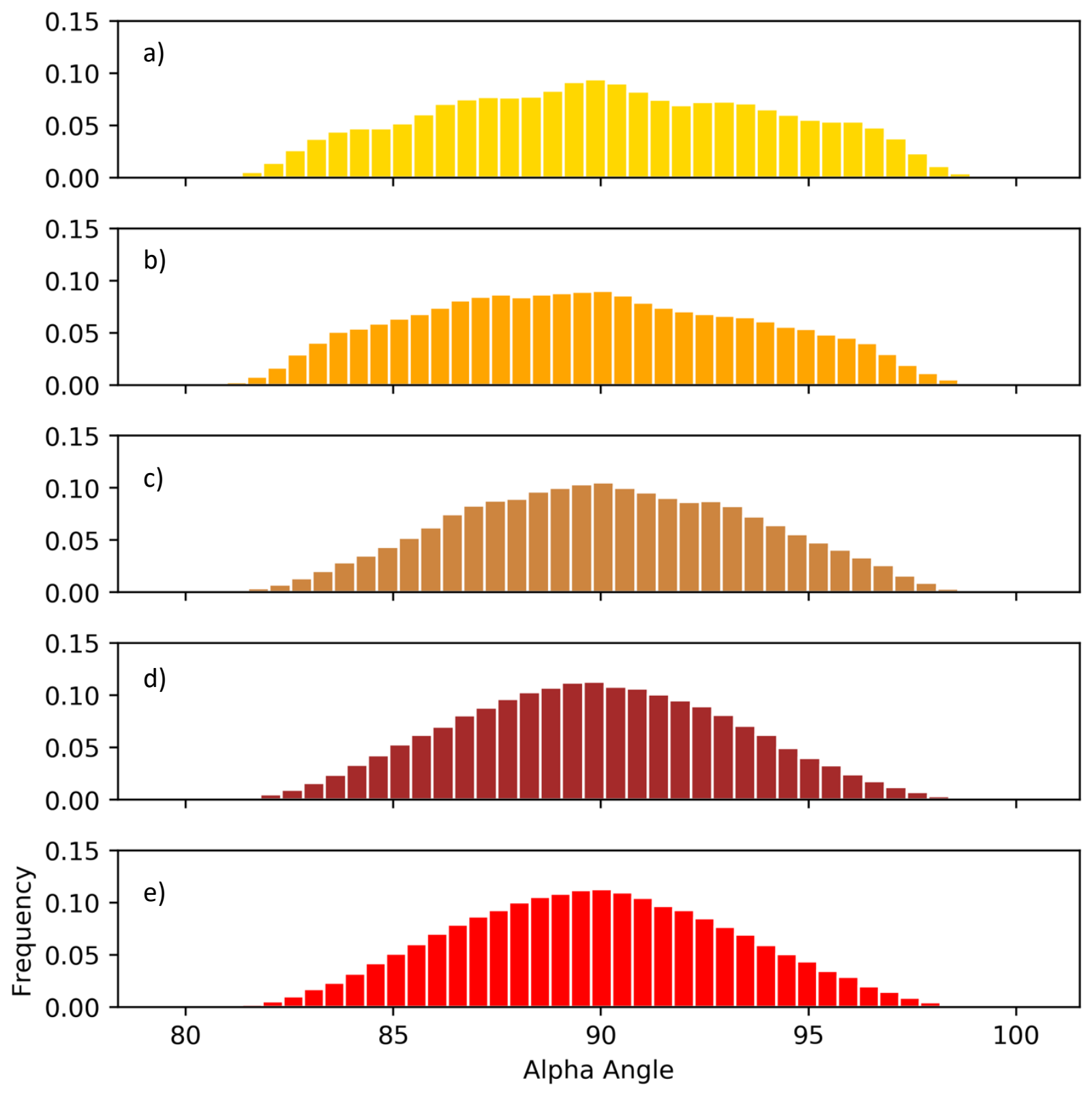

Figure S4 - Distribution of Alpha Angles throughout the trajectory for NPTF simulations at a) $200 \mathrm{~K}$, b) $225 \mathrm{~K}$, c) $250 \mathrm{~K}$, d) $275 \mathrm{~K} \mathrm{e)} 300 \mathrm{~K}$ 


\section{Comparison Free Energy Curves vs Larger Super-Cells}

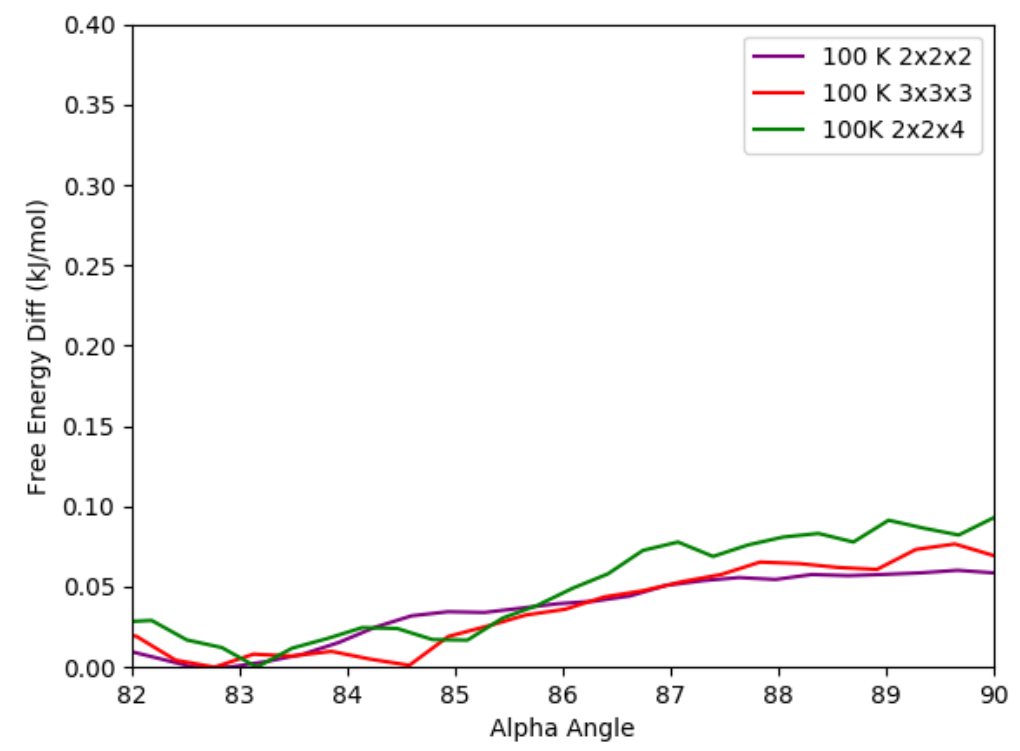

Figure S5. Comparisons of Free energy profiles from 100K Crystal-AFED simulations using a $2 \times 2 \times 2$ Super-Cell $(\mathrm{Th}=500 \mathrm{Tau}=2000)$ with a $2 \times 2 \times 4$ Super-Cell $(\mathrm{Th}=1500 \mathrm{Tau}=2000)$ and a 3x3x3 Super-Cell $(\mathrm{Th}=4000 \mathrm{Tau}=2000)$

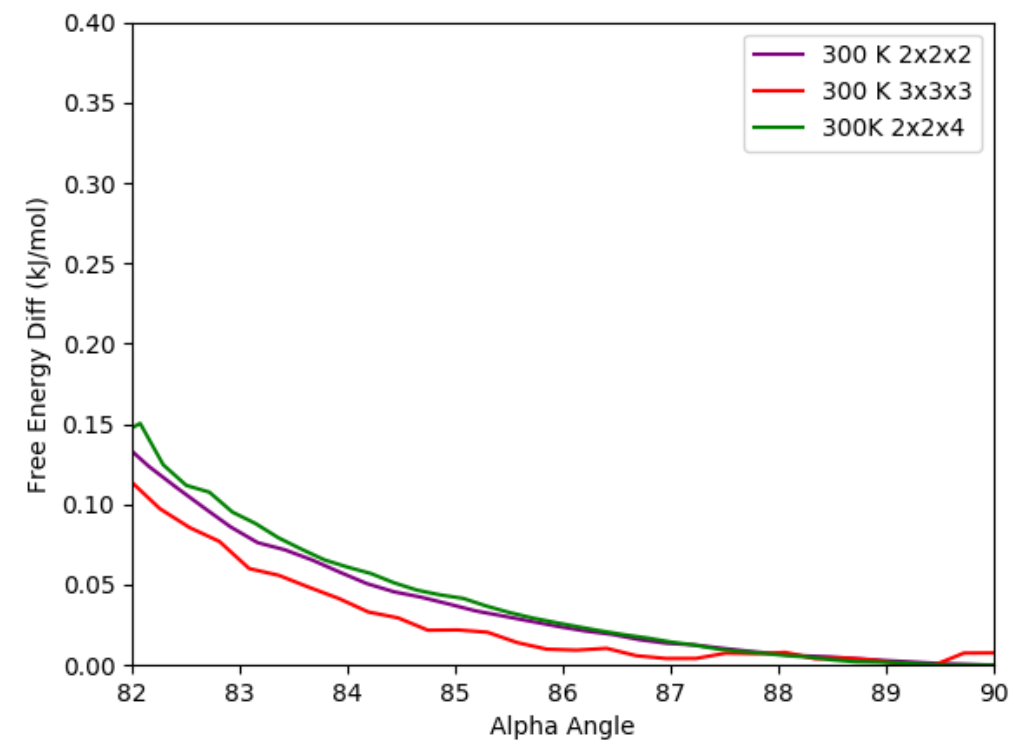

Figure S6. Comparisons of Free energy profiles from $300 \mathrm{~K}$ simulations using a 2x2x2 SuperCell (NPTF), using a 2x2x4 Super-Cell (NPTF), and a 3x3x3 Super-Cell $(\mathrm{Th}=2000 \mathrm{Tau}=$ 2000) 
Scatter Plot of Neighboring Layer Pair Displacements at 100K



Figure S7. Scatter plot of the layer center of mass displacements between layer pairs 1 and 2 at $100 \mathrm{~K}$ for the $100 \mathrm{~K}$ cAFED simulation 


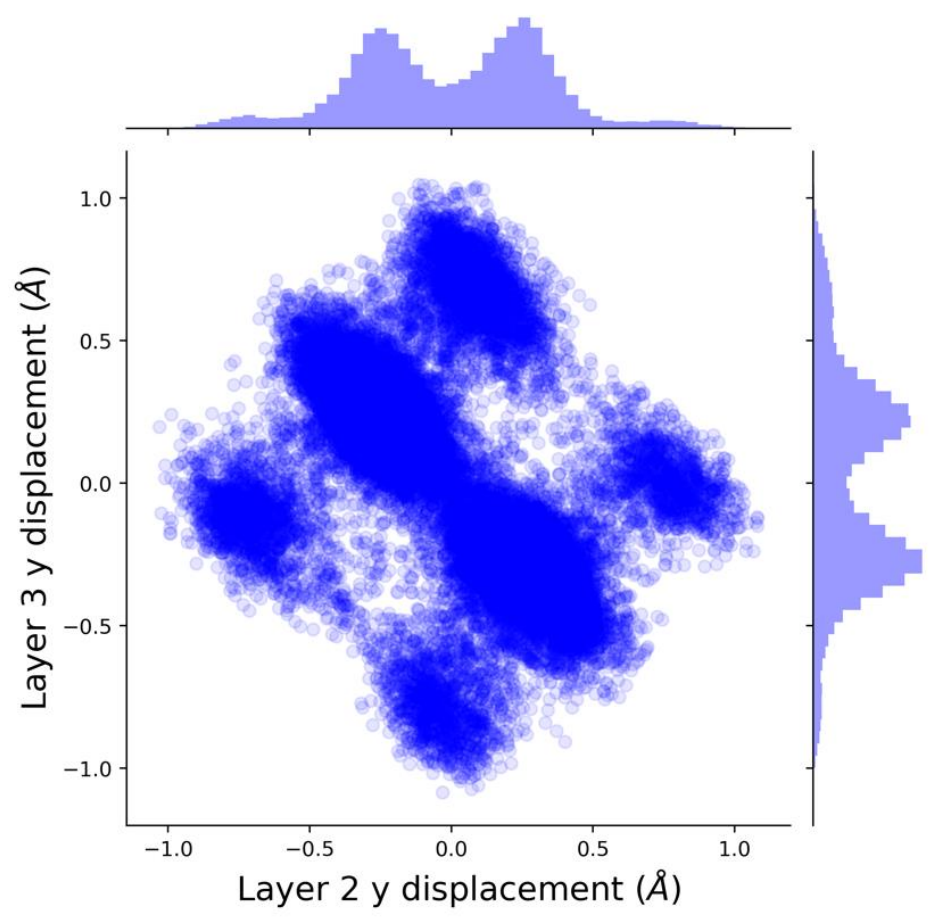

Figure S8. Scatter plot of the layer center of mass displacements between layer pairs 2 and 3 at $100 \mathrm{~K}$ for the $100 \mathrm{~K}$ cAFED simulation



Figure S9. Scatter plot of the layer center of mass displacements between layer pairs 3 and 4 at $100 \mathrm{~K}$ for the $100 \mathrm{~K}$ cAFED simulation 
Scatter Plot of Neighboring Layer Pair Displacements at 300K:

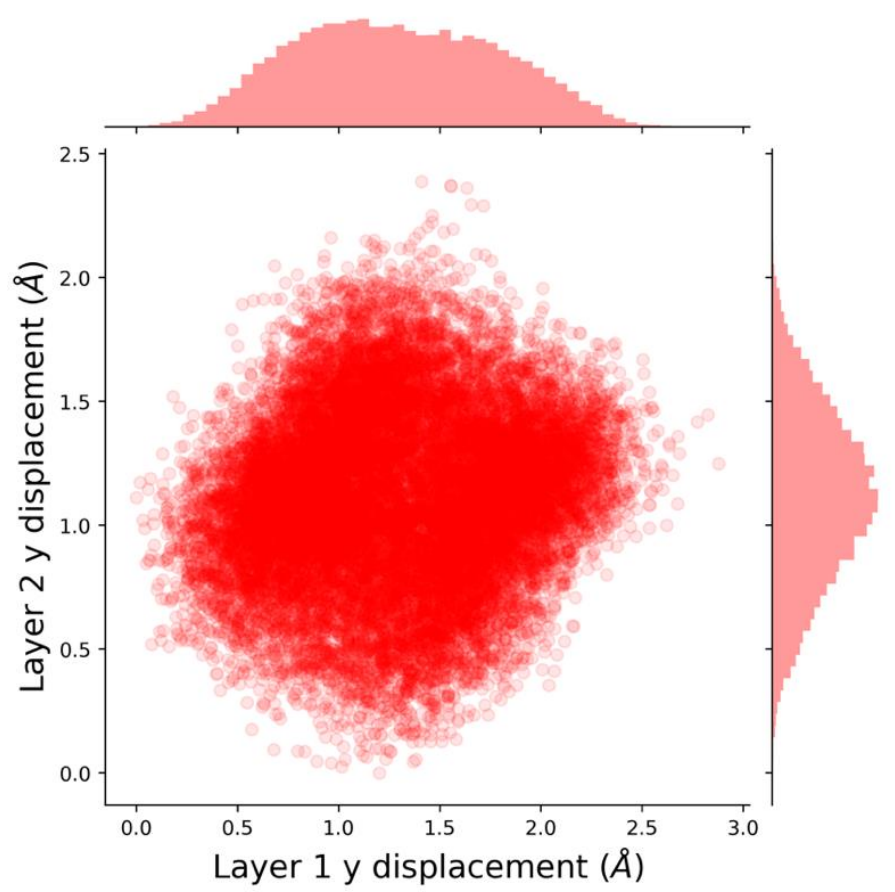

Figure S10. Scatter plot of the layer center of mass displacements between layer pairs 1 and 2 at $300 \mathrm{~K}$

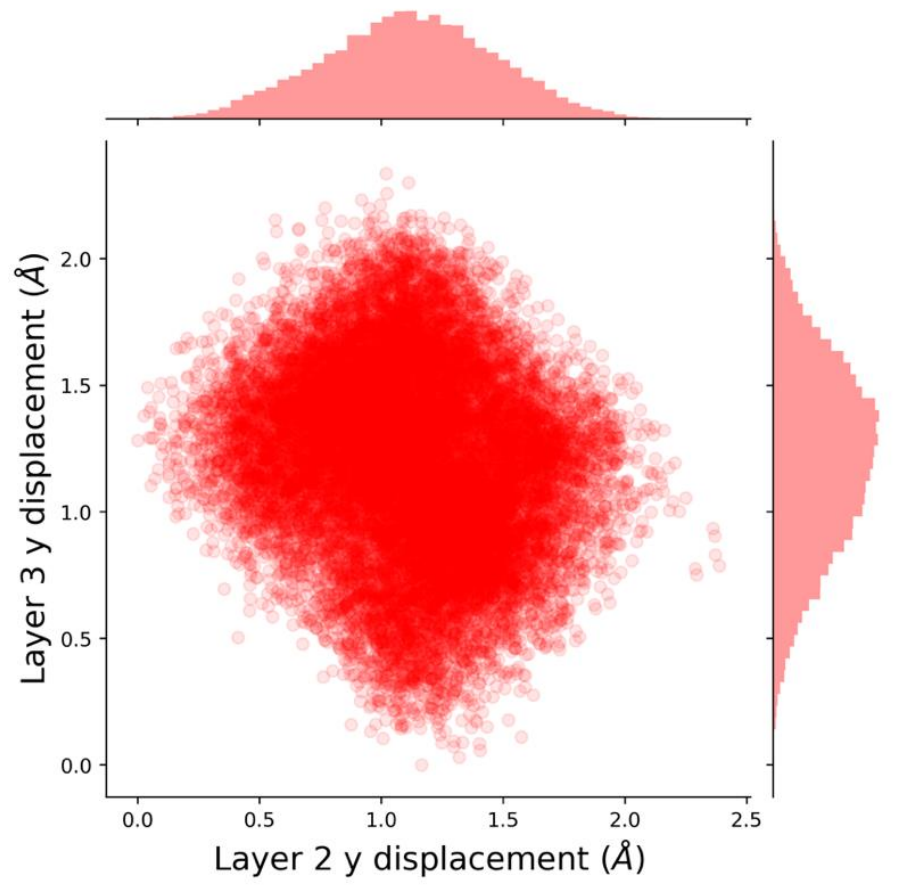

Figure S11. Scatter plot of the layer center of mass displacements between layer pairs 2 and 3 at $300 \mathrm{~K}$ 


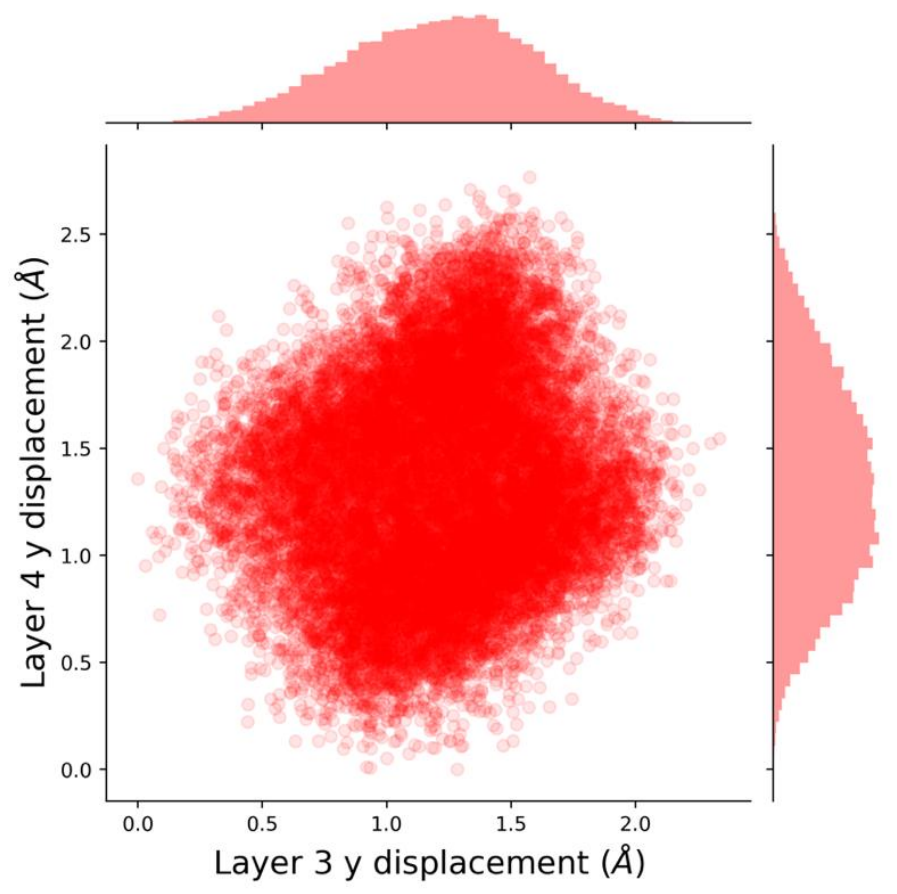

Figure S12. Scatter plot of the layer center of mass displacements between layer pairs 3 and 4 at $300 \mathrm{~K}$ 
Displacement Scatter Plots for 2x2x4 Super-Cell with many more layers for NonNeighboring Pairs at 100K
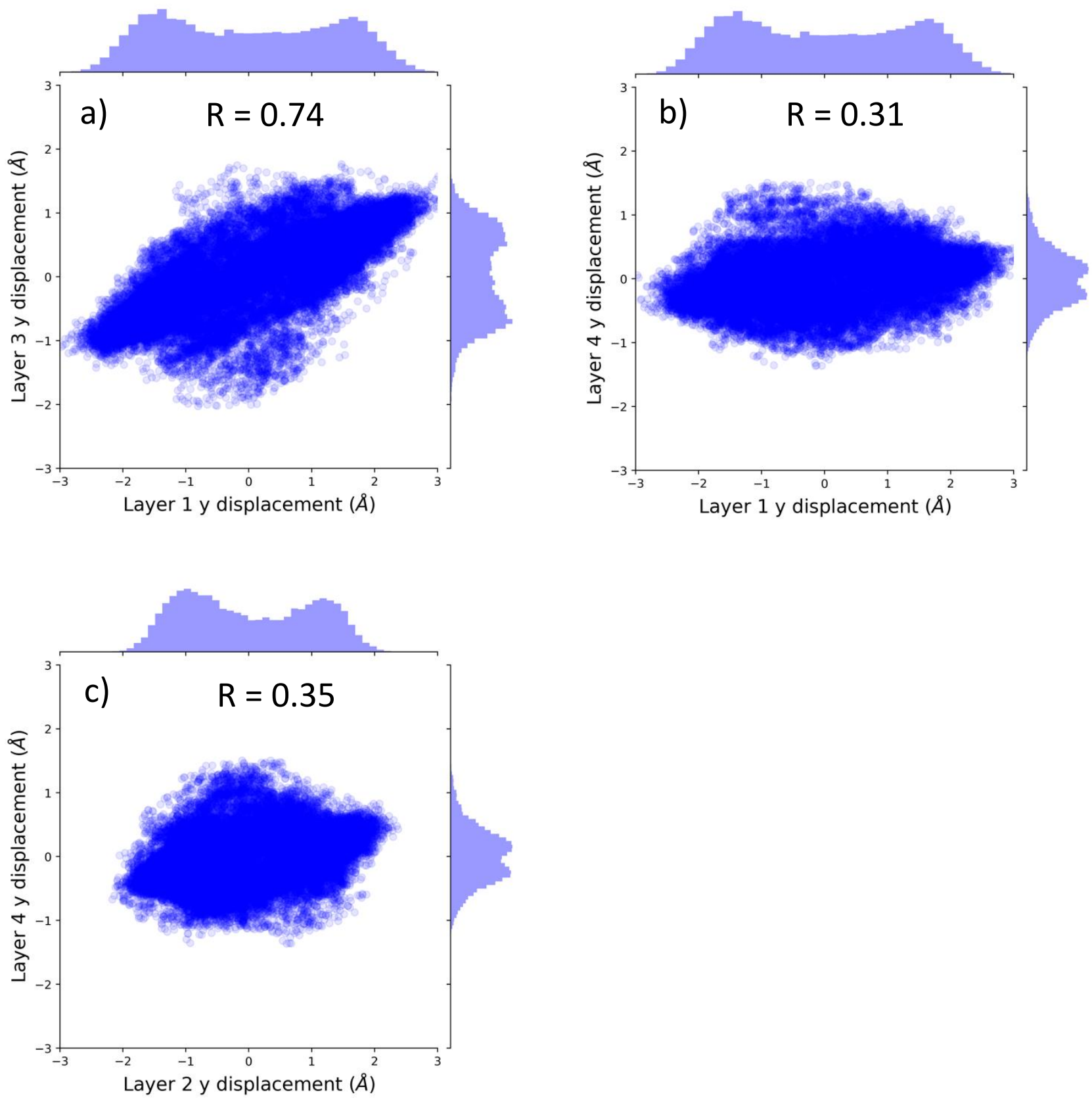

Figure S13. Comparisons of displacement scatter plots of a) layer pairs 1 and $3 \mathrm{~b}$ ) layer pairs 1 and 4 and c) layer pairs 2 and 4 for Crystal-AFED simulations at 100K for a 2x2x4 super-cell 
Displacement Scatter Plots for 2x2x4 Super-Cell with many more layers for NonNeighboring Pairs at 300K


Figure S14. Comparisons of displacement scatter plots of a) layer pairs 1 and $3 \mathrm{~b}$ ) layer pairs 1 and 4 and c) layer pairs 2 and 4 for Crystal-AFED simulations at $300 \mathrm{~K}$ for a $2 \times 2 \times 4$ super-cell 


\section{Determination of Layer Slip Measure}

- The centers of mass for each layer pair is first determined for each determined timeframe throughout the simulation

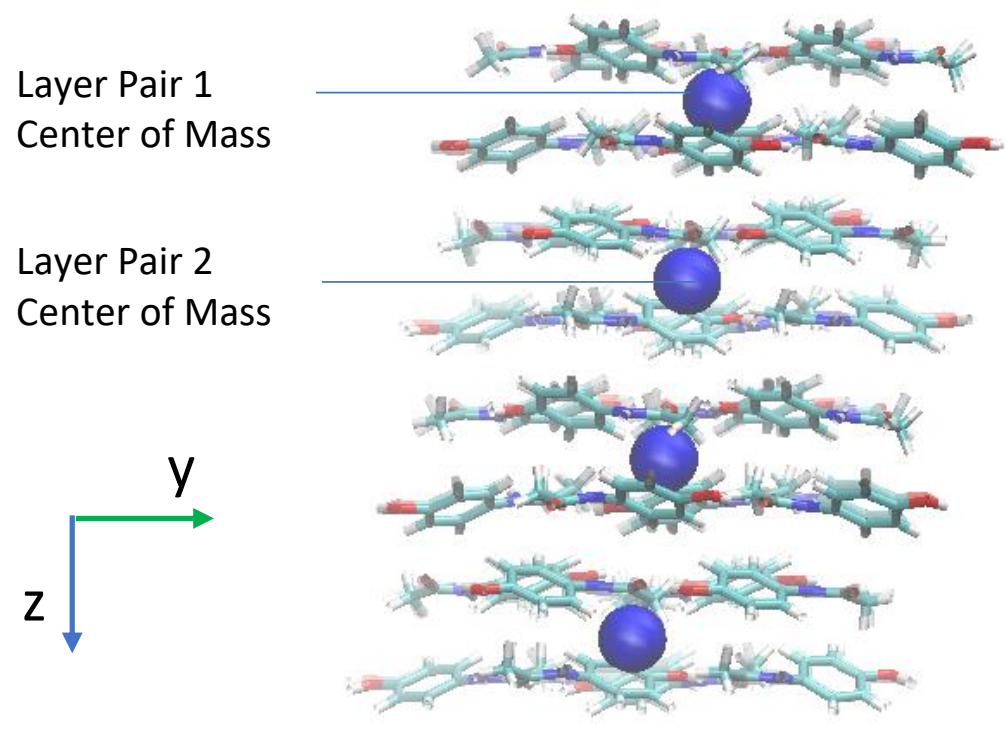

- The difference between the y coordinate of each layer pair center of mass is taken. (i.e. $\Delta Y 21-\Delta Y 23)$

- The absolute values of these differences are then summed up to determine a layer-slip measure. A value close to zero represents a perfectly aligned crystal whereas a higher value represents higher extents of layer slip disorder

$$
\begin{gathered}
\delta_{L S}=\left|\left(Y_{3}-Y_{2}\right)-\left(Y_{2}-Y_{1}\right)\right|+\left|\left(Y_{4}-Y_{3}\right)-\left(Y_{3}-Y_{2}\right)\right|+ \\
\left|\left(Y_{2}-Y_{1}\right)-\left(Y_{4}-Y_{3}\right)\right|+\left|\left(Y_{4}-Y_{1}^{\prime}\right)-\left(Y_{4}-Y_{3}\right)\right|+ \\
\left|\left(Y_{4}-Y_{1}^{\prime}\right)-\left(Y_{3}-Y_{2}\right)\right|+\left|\left(Y_{4}-Y_{1}^{\prime}\right)-\left(Y_{2}-Y_{1}\right)\right| \\
+\left|\left(Y_{4}-Y_{2}\right)-\left(Y_{3}-Y_{1}\right)\right| \\
\\
Y_{1}=\text { Layer Pair } 1 \text { COM } \\
Y_{2}=\text { Layer Pair } 2 \text { COM } \\
Y_{3}=\text { Layer Pair } 3 \text { COM } \\
Y_{4}=\text { Layer Pair } 4 \text { COM } \\
Y_{1}^{\prime}=\text { Layer Pair } 1 \text { Periodic Image COM }
\end{gathered}
$$




\section{Simulation Free Energy Comparisons Between Starting with III-o vs Starting with III-m}

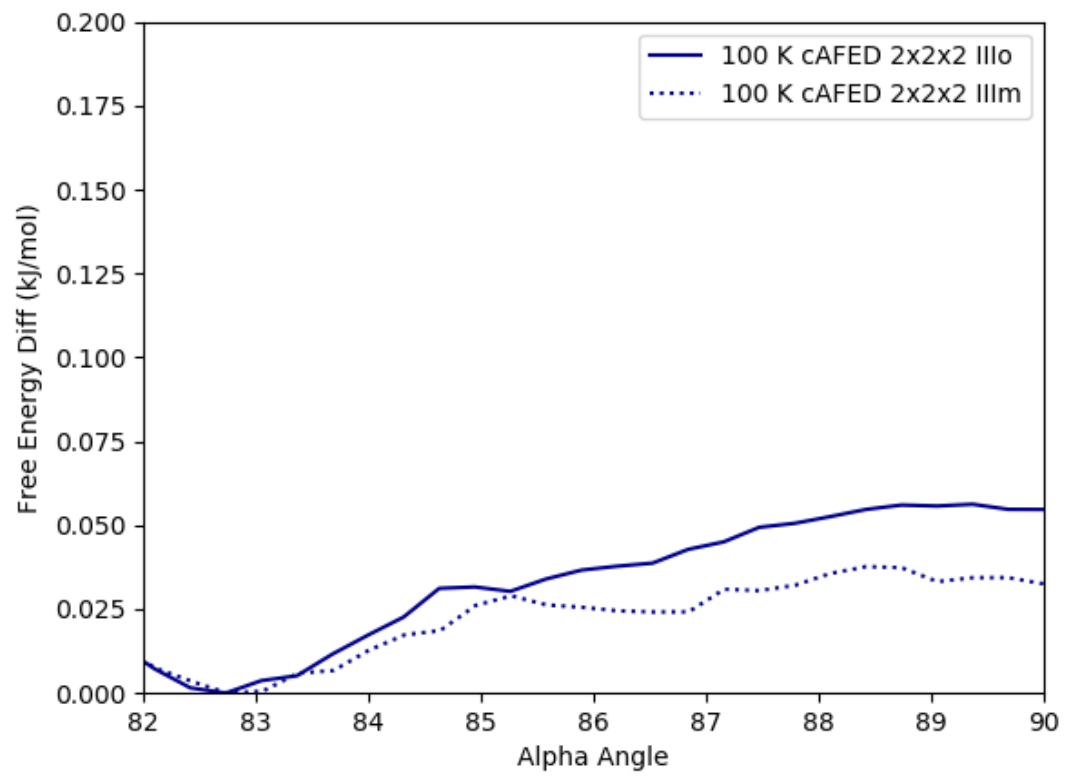

Figure S15. Comparisons of the folded free energy surface using Crystal-AFED with $\mathrm{Th}=500$ $\mathrm{K}$ and $\mathrm{Tau}=2000 \mathrm{~K}$ at a molecular temperature of $100 \mathrm{~K}$ starting with the orthorhombic and monoclinic Form III structures

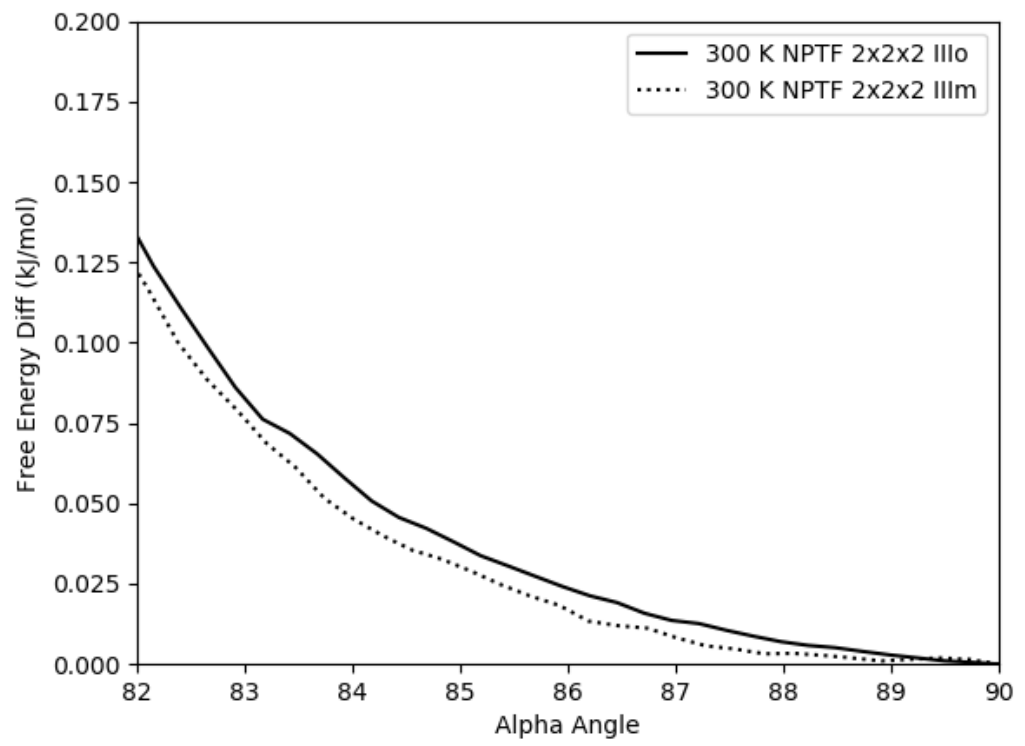

Figure S16. Comparisons of the folded free energy surface using NPTF at a molecular temperature of $300 \mathrm{~K}$ starting with the orthorhombic and monoclinic Form III structures 